\title{
Author Correction: Reconstruction of the human blood-brain barrier in vitro reveals a pathogenic mechanism of APOE4 in pericytes
}

Joel W. Blanchard, Michael Bula, Jose Davila-Velderrain, Leyla Anne Akay @i, Lena Zhu, Alexander Frank (1), Matheus B. Victor, Julia Maeve Bonner, Hansruedi Mathys, Yuan-Ta Lin, Tak Ko, David A. Bennett, Hugh P. Cam, Manolis Kellis $\mathbb{B}$ and Li-Huei Tsai (D)

Correction to: Nature Medicine https://doi.org/10.1038/s41591-020-0886-4, published online 8 June 2020.

In the version of this article initially published, the units used for TEER results $\left(\mathrm{ohm} \mathrm{cm}^{-2}\right)$ were incorrect in the third, fourth, fifth and final sentences of the fourth paragraph in the first subsection of Results; along the vertical axis of Fig. 1k; and in the fourth sentence of the second subsection of Methods ('iBBB permeability studies'). The correct units are 'ohm $\mathrm{cm}^{2}$. The error has been corrected in the HTML and PDF versions of the article.

Published online: 29 January 2021

https://doi.org/10.1038/s41591-021-01250-8

(c) The Author(s), under exclusive licence to Springer Nature America, Inc. 2021

\section{Author Correction: Tau molecular diversity contributes to clinical heterogeneity in Alzheimer's disease}

Simon Dujardin (1), Caitlin Commins, Aurelien Lathuiliere, Pieter Beerepoot $\mathbb{B}$, Analiese R. Fernandes, Tarun V. Kamath, Mark B. De Los Santos (D), Naomi Klickstein, Diana L. Corjuc, Bianca T. Corjuc, Patrick M. Dooley, Arthur Viode, Derek H. Oakley, Benjamin D. Moore, Kristina Mullin, Dinorah Jean-Gilles, Ryan Clark, Kevin Atchison, Renee Moore, Lori B. Chibnik, Rudolph E. Tanzi @ , Matthew P. Frosch, Alberto Serrano-Pozo@, Fiona Elwood, Judith A. Steen, Matthew E. Kennedy and Bradley T. Hyman (1)

Correction to: Nature Medicine https://doi.org/10.1038/s41591-020-0938-9, published online 22 June 2020.

In the version of this article initially published, in the legend to Extended Data Fig. 9, Extended Data Fig. 3a,b was incorrectly identified as Supplementary Fig. 4a,b, and Extended Data Fig. 6a,b was incorrectly identified as Supplementary Fig. 8a,b. The correct section of that legend is as follows: "...tau burden from Extended Data Fig. 6a,b (respectively a, and b) but also oligomeric tau levels from Fig. 3a (respectively c, and $\mathbf{d}, \mathrm{p}=0.057$ ) and 2 epitopes of tau hyperphosphorylation: PHF6, from Extended Data Fig. 3b (respectively e, and $\mathbf{f}$, $\mathrm{p}=0.051$ ) and AT8 from Extended Data Fig. 3a (respectively $\mathbf{g}$, and $\mathbf{h}$ ).... The error has been corrected in the HTML and PDF versions of the article.

Published online: 29 January 2021

https://doi.org/10.1038/s41591-021-01251-7

(c) The Author(s), under exclusive licence to Springer Nature America, Inc. 2021 